\title{
Diagnosis and Optimization of Interior Idle Sound Quality Based on Relative Approach
}

\author{
Su Lilii, a ${ }^{\text {*, Li Hongliang }}{ }^{2, b}$, Yang Chunqing ${ }^{3, c}$, Tian Yuzhu $^{4, d}$ \\ ${ }^{1}$ China Automotive Technology and Research Center, Tianjin, 300300, China \\ ${ }^{2}$ China Automotive Technology and Research Center, Tianjin, 300300, China \\ ${ }^{3}$ China Automotive Technology and Research Center, Tianjin, 300300, China \\ ${ }^{4}$ Hebei University of Technology, School of Mechanical Engineering, Tianjin,300132, China \\ asulili@catarc.ac.cn, ${ }^{b}$ lihongliang@catarc.ac.cn, cyangchunqing@catarc.ac.cn, ${ }^{\mathrm{d}} 1007901630 @ q q . c$ \\ om
}

Keywords: Vehicle Engineering; Sound quality; Idle condition; Relative approach

\begin{abstract}
Customers often complain a vehicle noise although the interior noise A-weighted sound pressure level has reached the same level of excellent vehicle. To solve the problem of large interior noise and messy sound of an own brand SUV, relative approach analysis is utilized, which is detected the frequency component perceived by human hearing easily. Then, used the method of separating the noise source, confirming and rectifying the noise source. Optimized test data show: A-weighted sound pressure level of the SUV interior noise in neutral idle condition was reduced from $43.86 \mathrm{~dB}(\mathrm{~A})$ to $37.5 \mathrm{~dB}(\mathrm{~A})$, reached the same level of joint-venture brand SUV outstanding level. Clutter sound disappeared, sound quality subjective evaluation value improved. Relative approach analytical method is sensitive to human hearing detected frequency band, therefore, using this method can quickly and accurately lock the noise source, effective solved the problem of mismatch between listening experience and sound pressure level.
\end{abstract}

\section{Introduction}

Interior noise environment can intuitively reflect the ride comfort and the quality of the vehicle, so vehicle sound quality has become an important factor of customers purchasing [1]. Idle as a typical working condition, $\mathrm{NVH}$ performance of the vehicle is most directly reflected by the interior noise [2]. A-weighted sound pressure level is the conventional evaluation standard of sound. However, A-weighted sound pressure level has reached the better than average level of the same type vehicle, but the customers still feel the interior noise unbearable, on the contrary, sometimes A-weighted sound pressure level of interior noise, which is larger than it, is more pleasing to the customers, instead of feeling more acceptable. This is due to difference of each vehicle sound quality. Sound quality reflects people subjective feeling of sound in certain conditions of vehicle. The human ear is very difficult to detect the change when sound pressure level within $3 \mathrm{~dB}(\mathrm{~A})$, so clutter sounded even more frustrating when idle noise under $40 \mathrm{~dB}$ (A) [3]. It appears to have little effect to blindly reduce the interior sound pressure level, but should pay more attention to the purity of the sound and improving sound quality.

Aiming at the problem of a self-owned brand SUV louder and clutter interior noise on idle condition, relative approach analysis is used to calculate the idle condition interior noise with HEAD Artemis software, it has the advantage that can get the special frequency band which human ear to detect easily. In order to determine these special frequency band, separating noise source one by one, focusing and improving. This method can quickly find the greatest contribution of noise source accurately which influencing interior sound quality, not only solved mismatch between Auditory feel and sound pressure level, but also effectively control the vehicle development cycle and cost. 


\section{Psychoacoustics parameters.}

Acoustic analysis method in common use such as Level vs. Time, FFT, or 1 / n Octave, just embodies the sound energy distribution of time domain and frequency domain. Signal usually carries information of euphoria or anxious, but the above several ways can't explain the auditory information. Psychological acoustics (Psychoacoustics), combining physiology and psychology, establishes a mapping relationship between the sound event and the auditory event. Psychoacoustics with quantitative manner reflects what happens in a sound hearing feeling. Psychoacoustics parameters include loudness, sharpness, relative approach, articulation index, roughness, fluctuation strength, tone, etc. [3].

Relative to the steady-state sound signal, the person's audition is especially sensitive to the signal changing over time. Auditory correlation (Relative Approach) is the mutation components of acoustic waves, which can be used as quantitative parameters of rattle and squeak. The basic algorithm is based on the template of the auditory Regression Analysis (Regression Analysis). The Regression Analysis can be respectively carried on in the time domain and frequency domain [4]. As shown in figure 1 , figure 2 , the horizontal axis represents the test time (s), the ordinate represents frequency $(\mathrm{Hz})$, color bars represent the amount of hearing related $(\mathrm{dB}(\mathrm{A}))$, only at the beginning of the sine and sweep sound signal, people ear has the most sensitive reaction. During a period of time, auditory stimulus for the two sound reception has leveled off. The dissonant feeling of interior noise is the result of sound signal instability or the obvious differences of noise frequency, so this article use Relative Approach to find the frequency of the interior noise which is the most susceptible to ear perception.

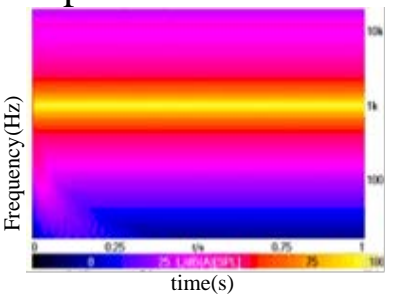

(a) $1 / 3$ octave

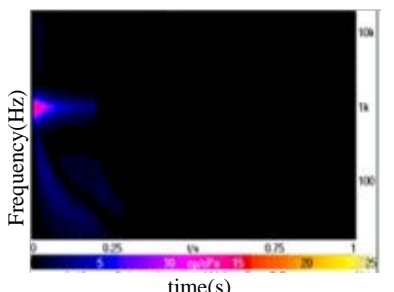

(b)Relative Approach

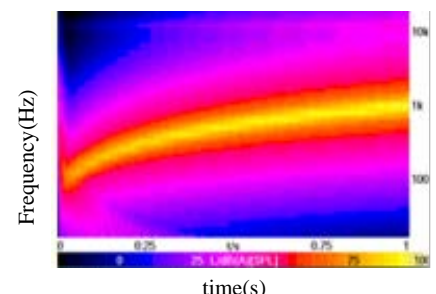

(a) $1 / 3$ octave

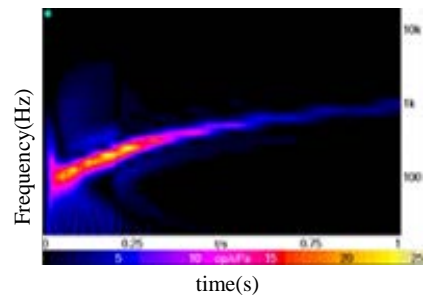

(b)Relative Approach

Fig 1. Sine signal

Fig 2. Sweep signal

\section{Idle interior noise source identification}

\subsection{Perceived noise frequency identification}

The car interior noise level on idle condition as shown in figure 1, the SPL (sound pressure level) of driver's right ear is $43.86 \mathrm{~dB}(\mathrm{~A})$, higher than the target value of the car about $6 \mathrm{~dB}$ (A), subjective evaluation group members reflect that the car sound make people feel dissonant, at the same time there is a clear "da da" sound. the sound quality subjective evaluation test score of 5 qualified (10 points system, 6 points are qualified). As can be seen from the figure 3, noise energy mainly concentrates in the $120 \sim 300 \mathrm{~Hz}$ low frequency band, but figure 4 shows the driver's right ear hearing related quantity to reflect. Near $200 \mathrm{~Hz}$ frequency will cause attention to the human ear, and another $1000 \mathrm{~Hz}$ sound is also sensitive for the human ear. 


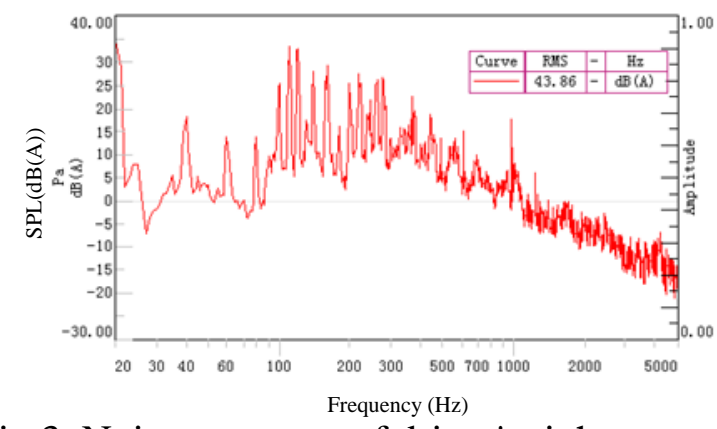

Fig 3. Noise spectrum of driver's right ear on the condition of idle AC off

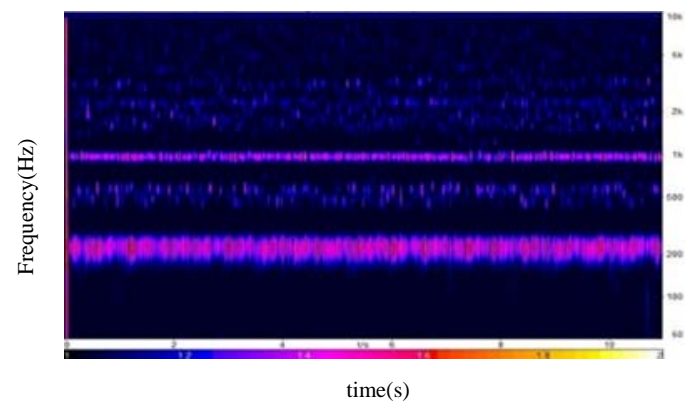

Fig 4. Relative approach of driver's right ear on the condition of idle AC off

\subsection{Noise source identification}

Idle noise contribution analysis experiment, the test data shows that radiation in powertrain ontology, the structure of the suspended pads pass noise and exhaust radiation noise, the structure of the steering pipe, air conditioning pipeline vibration of multiple noise sources, the greatest contribution of fuel oil system. As shown in figure 5, in the amount of idle noise contribution analysis experiments, driver's right ear noise separation from fuel line connected to the car in front of the $41.35 \mathrm{~dB}$ (A) reduce to $38.86 \mathrm{~dB}$ (A), the peak between $120 \sim 300 \mathrm{~Hz}$ is decreased obviously. To illustrate that the structure-borne noise caused by fuel line knocking the body need improvement.

Adjust external regulated power supply to the idle speed fuel pump working voltage, the stall condition of pump power supply test pump body radiation noise contribution to the car, the test data display (figure 6), the peak near $1000 \mathrm{~Hz}$ is caused by the radiation noise of fuel pump.

— Driver's right ear noise before fuel line separated from body

_ Fuel line and the car driver's right ear noise after separation

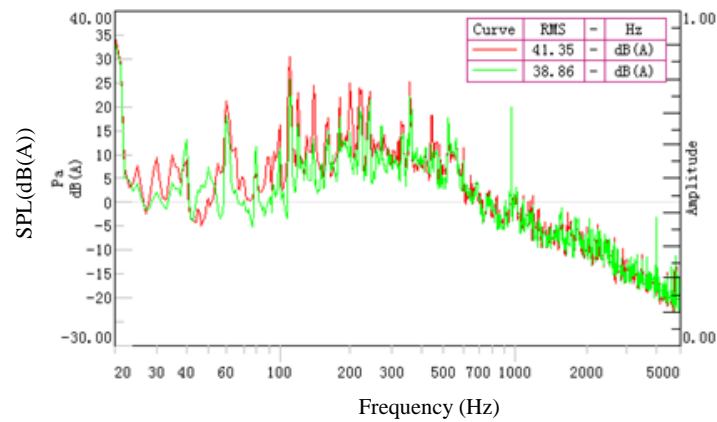

Fig 5. Fuel line connected to the car interior noise before and after separation
— The original state of driver's right ear noise

— Fuel pump driver's right ear noise external power supply situation

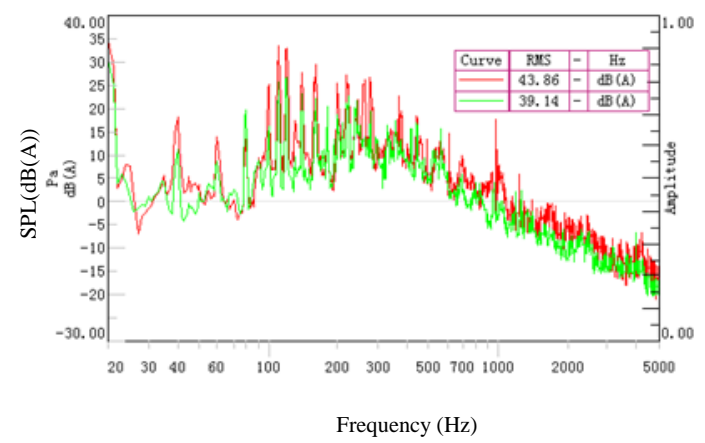

Fig 6. Fuel pump body radiation noise contribution amount

To quickly test the fuel system's contribution to the interior noise, first of all to inject air in the fuel line, because the oil and air compression ratio is different, which reduces fuel pulse and fuel piping vibration sensors according to $120 \sim 300 \mathrm{~Hz}$ vibration is reduced, and then use lead leather cover pump installed shielding pump radiation noise, peak near $1000 \mathrm{~Hz}$. Idle noise is reduced to $39.14 \mathrm{~dB}(\mathrm{~A})$ from $43.86 \mathrm{~dB}(\mathrm{~A})$, as shown in figure 2.

As a result, $120 \sim 300 \mathrm{~Hz}$ and $1000 \mathrm{~Hz}$ frequency noises of the vehicle idle noise, which are sensitive of the human ear, are respectively the structure-borne noise of fuel pulse and the radiation noise of fuel pump body.

\section{Verify the improvement measures and systems}

The methods reducing the structure-borne noise of fuel pulse are: (1) increasing pipeline diameter; (2) shorten the pipe length; (3) reduce pipeline curvature; (4) use the rubber soft way; (5) increases 
fuel pulsation damper; (6) fuel line with damping pad with increased body connection pipe clamp. Methods (1) (5) is to reduce excitation method, and (6) is the method of the performance improvement through the transmission path $[5,6]$.

In view of project development cycle and cost limit method (1) (4), 6 due to involve the fuel line to design and spatial layout, do not have the rectification conditions, so the scheme (5) for rectification fuel line oil pulse noise.

Pulsation damper is a kind of pressure vessel, which is used to eliminate the pressure fluctuation or flow pulsation in the pipe. It can stabilize the fluid pressure and flow, eliminate pipe vibration, protect downstream instrument and equipment, and increase pump volume efficiency. According to the principle, pulsation dampers can be classified to the airbag type and no moving part type. Airbag type fuel pulse dampers depend on the contraction and expansion of inert compressed gas to absorb liquid pressure or flow pulsation, which is suitable for the application of pulse frequency less than $7 \mathrm{~Hz}$; No moving parts type pulsation dampers use solid media direct interception fluid to buffer the effect of the pressure pulsation or flow pulsation, which is applied to weaken or eliminate the high frequency pulse. The latter is used as a performance optimization measure in view of the noise concentrating in the $120 \sim 300 \mathrm{~Hz}$.

Fuel pulsation damper (as shown in figure 8) is increased in the rear of fuel trail. After the optimization design, the interior noise of $120 \sim 300 \mathrm{~Hz}$ frequency band depresses remarkably (as shown in figure 9). The vibration accelerations of fuel line and the car body front surface vibration joint point reduce obviously, especially $Z$ direction vibration acceleration reducing from $0.11 \mathrm{~m} / \mathrm{s}^{2}$ to $0.03 \mathrm{~m} / \mathrm{s}^{2}$ (as shown in figure 10). These prove that fuel pulsation damper is effective for reducing the structure-borne noise caused by fuel pulsation in the fuel line.

_ The original state of driver's right ear noise

— The fuel system test driver's right ear noise

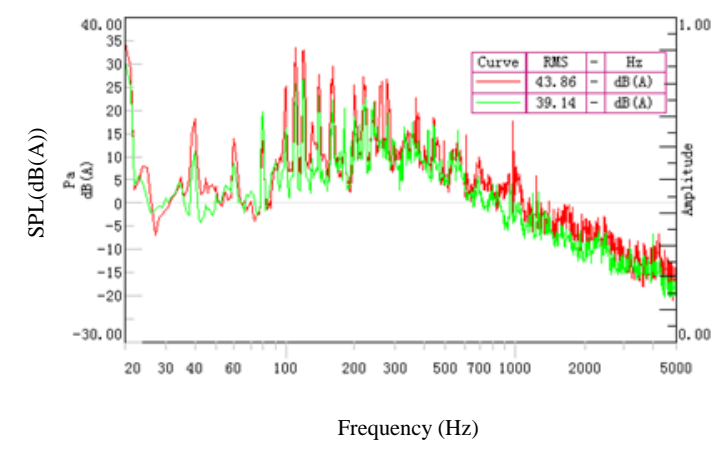

Fig 7. Fuel system problem noise contrast before and after confirmation

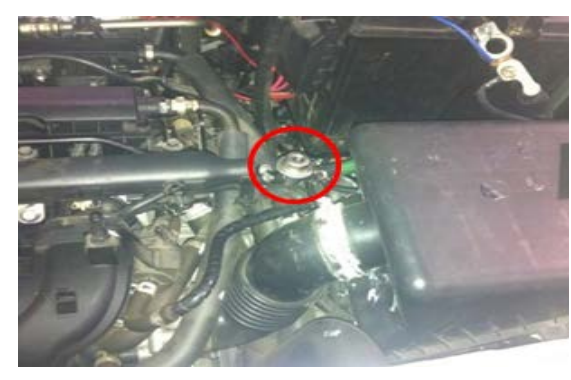

Fig 8. Fuel pulsation dampers

Fuel pump was replaced with metal material. Peak near $1000 \mathrm{~Hz}$ noise is reduced, improving the sound insulation performance can effectively block fuel pump body radiation noise.

Gathers the interior noise of fuel system after rectification again relative approach analysis and calculation, and subsequent radiation for hydraulic steering noise and exhaust noise, the noise of the suspended structure such as rectification, until the sound pressure level to meet goals and objectives. After the sound pressure level standard car idle noise is feel noise at the launch of the subjective evaluation test, purer, the sound quality subjective evaluation score of 7 points. Test data is shown in figure 11 , the driver right ear noise is decreased from $43.86 \mathrm{~dB}(\mathrm{~A})$ to $37.5 \mathrm{~dB}(\mathrm{~A})$, "CATARC SOUND" harshness (NVH) database performance, according to the data the excellent level has reached the same level joint SUV. Compared relative approach before with after optimization (figure 12), the sensitive frequencies of human hearing are obviously depressed. 
- Driver's right ear noise before fuel system optimization

— The fuel system optimized driver's right ear noise

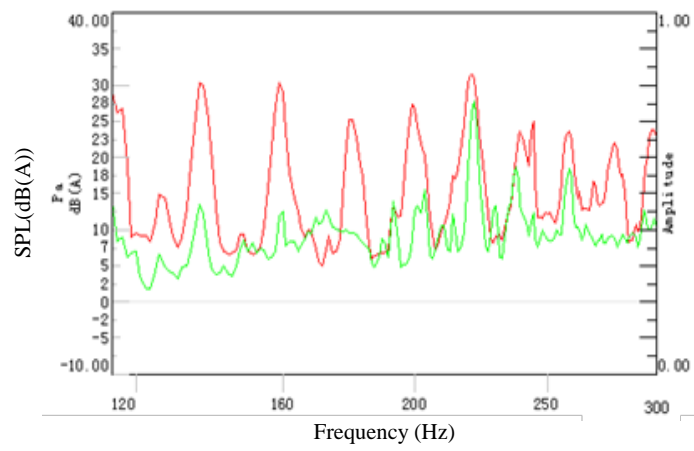

Fig 9. Driver's right ear noise before and after optimization $(120 \sim 300 \mathrm{~Hz})$
— Fuel system to optimize fuel line and body before installing vibration

— Fuel system optimized fuel line with body installation point vibration

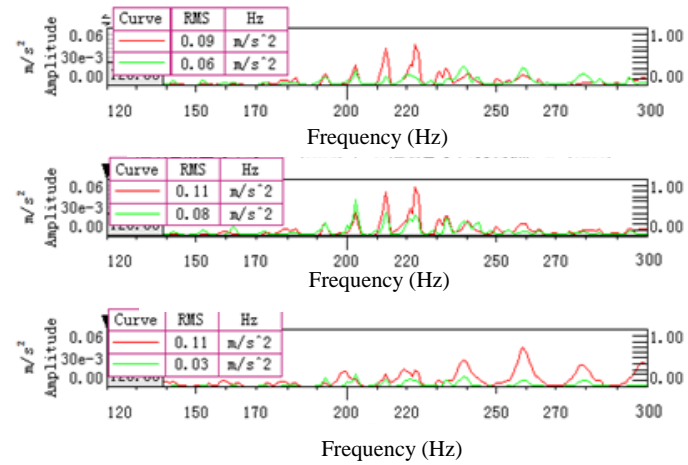

Fig 10. Fuel line before and after optimization and the small car body vibration installation points

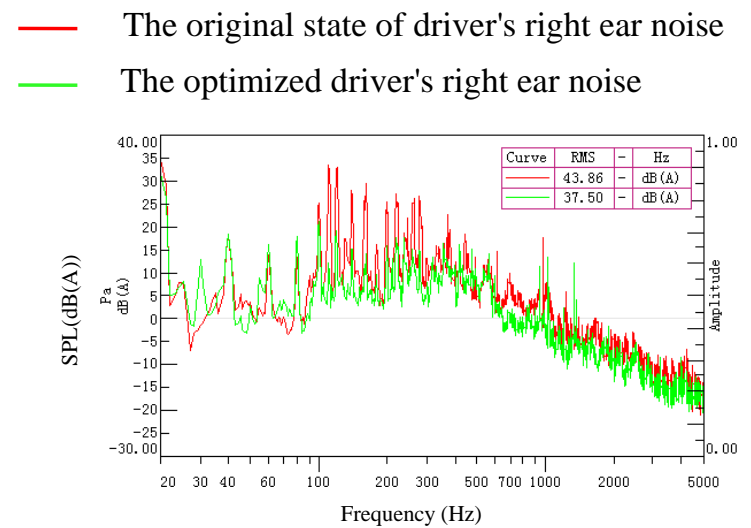

Fig 11. Idle noise before and after the rectification

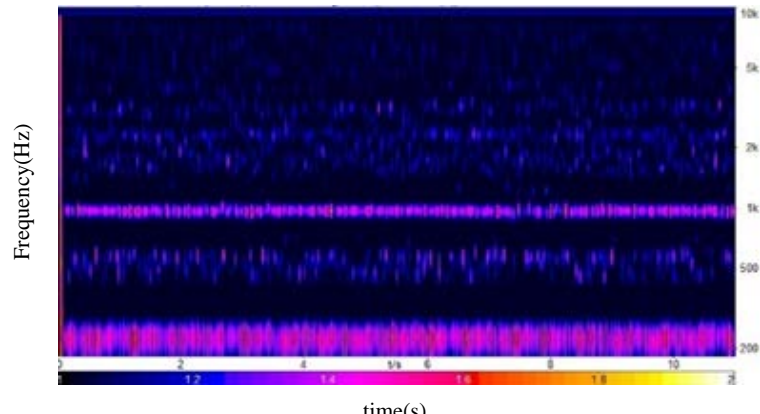

(a). Before optimization

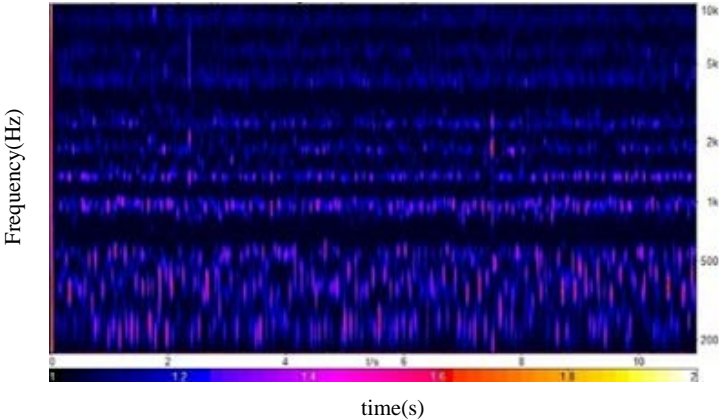

(b). After optimization

Fig 12. Performance driver's right ear hearing related amount before and after

As shown in table 1, the gap of improvement between before and after is obvious, for the driver's ear position idle noise, loudness, sharpness and relative approach have a strong correlation with sound quality subjective evaluation results, these three parameters are greatly reduced after improvement, and subjective evaluation results measured by jury increased from 5.5 to 7 points. 
Table 1 Psychoacoustics parameters comparison before and after improvement

\begin{tabular}{ccc}
\hline Evaluation parameters- & before optimization & after optimization \\
\hline SPL (dBA) & 44.4 & 37.5 \\
\hline Loudness (sone) & 3.39 & 2.25 \\
\hline Sharpness (acum) & 0.647 & 0.665 \\
\hline Roughness (asper) & 0.356 & 0.249 \\
\hline Fluctuation strength (vicil) & 0.0178 & 0.0201 \\
\hline Articulation Index (\%) & 98 & 99.7 \\
\hline Relative approach (dB(A)) & 21.6 & 16.6 \\
\hline Subjective evaluation results (point) & 5.5 & 7 \\
\hline "CATARC SOUND" NVH performatce database shows thatter the performance improvements,
\end{tabular}

"CATARC SOUND" NVH performance database shows that after the performance improvements, this SUV idling noise is below average of 11 SUV vehicles in the same level and price range (Table 2).

Table 2 "CATARC SOUND" database data of 11 SUV vehicles in the same level and price range

\begin{tabular}{ccc}
\hline & own brand (5) & Joint venture brand (6) \\
\hline $\mathrm{SPL}(\mathrm{dBA})$ & 40 & 38 \\
\hline
\end{tabular}

\section{Summary}

To solve the problem of large interior noise and messy sound of an own brand SUV, relative approach analysis is utilized, which is detected the frequency component perceived by human hearing easily. Then, used the method of separating the noise source, confirming and rectifying the noise source. According to the above process, determining the noise source of most sensitive to human hearing are fuel system, hydraulic steering, exhaust radiated noise, etc., Optimized test data show: A-weighted sound pressure level of the SUV interior noise in neutral idle condition was reduced from 43.86dB(A) to 37.5dB(A), reached the same level of joint-venture brand SUV outstanding level. Clutter sound disappeared, sound quality subjective evaluation value improved. Relative approach analytical method is sensitive to human hearing detected frequency band, therefore, using this method can quickly and accurately lock the noise source, effective solved the problem of mismatch between listening experience and sound pressure level.

This paper adopted by the diagnostic method, noise recognition process can be spread to the interior noise of a similar problem.

\section{Acknowledgement}

In this paper, the research was sponsored by the Tianjin Key Project (Project No. 14TXSYJC00457).

\section{References}

[1]. Su Lili. Interior sound quality subjective and objective evaluation and control method research [D]. Jilin: jilin university, 2012.

[2]. Da-liang Chen, hong-liang li, CheYunLong. Passenger car interior noise source identification in idle speed, the control measures study [J]. Journal of automotive technology. 2014 (7): $1 \sim 5$

[3]. PangJian. Car body noise and vibration control [M]. Beijing: mechanical industry publishing house.

[4]. 2015HEAD ArtemiS Help[Z]

[5]. Huang Wenbing, rob, Wang zhu tong. Idle car interior noise test significantly analysis and control [J]. Journal of automotive technology, 2014 (11): 7 10

[6]. Lee soldier. Idle interior noise analysis and control method research [J]. Mechanical engineers, 2015 (4): $149 \sim 152$ 